\title{
RELATIVE PROJECTIVITY, THE RADICAL AND COMPLETE REDUCIBILITY IN MODULAR GROUP ALGEBRAS
}

BY

\author{
D. C. KHATRI
}

ABSTRACT. If $H \leq G$ and every $G$-module is $H$-projective then $(G, H)$ is a projective pairing. If $\operatorname{Rad} F G \subseteq(\operatorname{Rad} F H) F G$ then $(G, H)$ is said to have property $\rho_{0} . A$ third property considered is that for each irreducible $H$-module the induced $G$-module be completely reducible. It is shown that these three are equivalent properties in many interesting cases. Also examples are given to show that they are, in general, independent of each other.

Let $H$ be a subgroup of a finite group $G$ and $F$ a field of characteristic $p>0$. The $H$-projective $F G$-modules were studied by Hochschild [6] and D. G. Higman [5]. Green [4] used this concept to classify indecomposable FG-modules through the theory of vertices and sources. In an earlier paper [7] we characterized the class of these subgroups $H$ of $G$ for which every $F G$-module is $H$-projective. In this case we call $(G, H)$ a projective pairing. As was shown in the last part of [7], a certain property (property $\rho$ : See Definition 3) of radicals of group algebras of $G$ and $H$ is closely connected with this concept. As it turns out, another property-the complete reducibility of induced modules, induced from an irreducible $\mathrm{FH}$-module-is almost equivalent to the two properties mentioned above. In this paper we give some sufficient conditions under which the three properties mentioned above are equivalent. We also show by means of an example that in general projective pairing does not imply property $\rho$.

2. Preliminary definitions and results. Let $F$ be a field of characteristic $p>0$ and $G$ a finite group such that $p$ divides $|G|$, the order of $G$. All modules under consideration will be right modules, finitely generated over $F$. Let $\Re$ be an FG-module, $F G$ being the group algebra of $G$ over $F$, and let $H$ be a subgroup of $G$. By $\mathbb{R}_{H}$ we denote $\mathbb{N}$ considered as $F H$-module in a natural way. Similarly, if $\Re$ is a $F H$-module, the induced $F G$-module is denoted by $\Re^{G}=\Re \otimes_{F H} F G$, where $\otimes$ denotes the tensor product. For standard properties of induced modules we refer to Curtis and Reiner [3], or Green [4].

Definition 1. Let $H$ be a subgroup of $G$. An FG-module $\mathbb{N}$ is said to be $H$ projective if every exact sequence $0 \rightarrow \Re \rightarrow L \rightarrow \Re \rightarrow 0$ of $F G$-modules for which

Received by the editors May 8, 1972.

AMS (MOS) subject classifications (1970). Primary 20C05; Secondary 16A26.

Key words and phrases. Projective pairing, Jacabson radical, complete reducibility, Frobenius groups. 
the associated sequence of restrictions $0 \rightarrow \Re_{H} \rightarrow L_{H} \rightarrow \Re_{H} \rightarrow 0$ splits over FH is itself split over $F G$.

Definition 2. Let $H<G$. We say that $(G, H)$ is a projective pairing if every exact sequence $0 \rightarrow \Re \rightarrow L \rightarrow \Re \rightarrow 0$ of $F G$-modules for which the exact sequence $0 \rightarrow \Re_{H} \rightarrow L_{H} \rightarrow \Re_{H} \rightarrow 0$ splits over $F H$ is itself split over $F G$.

It follows immediately that $(G, H)$ is a projective pairing if and only if every $F G$-module is $H$-projective.

Definition 3. Let $G=\bigcup_{i=1}^{k} H x_{i}, x_{1}=1$, be a coset decomposition of $G$ over H. Treating $F G$ as free module over $F H$ with basis $\left\{x_{1}, x_{2}, \ldots, x_{k}\right\}$ we say that $(G, H)$ has property $\rho$ over $F$ if, whenever $\Sigma_{i} p_{i} x_{i} \in \operatorname{Rad} F G, p_{i} \in F H$, then each $p_{i} \in \operatorname{Rad} F H$, where $\operatorname{Rad}$ denotes the Jacobson radical of the ring concerned.

In [9] it is shown that property $\rho$ is independent of the choice of coset representatives and that it is a transitive property in the sense that if $K \subseteq H \subseteq G$ and the pairs $(G, H)$ and $(H, K)$ have property $\rho$ then $(G, K)$ has property $\rho$.

It is evident that property $\rho$ is equivalent to the requirement $\operatorname{Rad} F G \subseteq$ $(\operatorname{Rad} F H) F G$.

The following cirteria of $H$-projectivity are due to Higman [5] and Green [4]:

(2.1) The following statements for an FG-module $\Re$ are equivalent:

(i) $\mathfrak{M}$ is $H$-projective.

(ii) $M$ is a component (i.e. is isomorphic to a direct summand of $\left(\mathbb{M}_{H}\right)^{G}$.

(iii) There exists an $F H$-module $\Re$ such that $\mathbb{R}$ is a component of $\mathfrak{R}^{G}$.

(iv) There exists an $F H$-endomorphism $\eta$ of $\mathbb{N}$ such that $\Sigma_{i} x_{i}^{-1} \eta x_{i}=1_{\mathscr{P}}$ where $\left\{x_{i}\right\}$ are the coset representatives of $G$ over $H$ and $1_{m}$ is identity mapping over $\Re$.

We list the following two results of [7] which we will use frequently:

(2.2) If $K \subseteq H \subseteq G$ and $(G, H)$ and $(H, K)$ are projective pairings, then $(G, K)$ is a projective pairing.

(2.3) $(G, H)$ is a projective pairing if and only if $H$ contains a $p$-Sylow subgroup of $G$.

Definition 4. We define the following classes of subgroups of $G$ :

$\mathscr{P}=\mathscr{P}(G, F)=\{H<G \mid(G, H)$ is a projective pairing over $F\}$,

$R=R(G, F)=\{H<G \mid(G, H)$ has property $\rho$ over $F\}$,

$\mathcal{C}=\mathcal{C}(G, F)=\{H<G \mid$ for each irreducible $F H$-module $\Re$, the induced $F G$ module $\mathfrak{R}^{G}$ is completely reducible $\}$.

Note that here $H<G$ means $H$ is a proper subgroup of $G,\{1\}$ being considered as proper subgroup.

Also the following two results of D. A. R. Wallace ([12], [13]) will be important to our discussion:

(2.4) If $|G|=p^{a} m,(p, m)=1$ then $\operatorname{dim}_{F}(\operatorname{Rad} F G) \geq p^{a}-1$; the equality 
holds if and only if $G=P M, M \triangle G, P \cap M=1$ is a Frobenius group with kernel $M$ and complement $P$, a $p$-Sylow subgroup of $G$; $p$ being the characteristic of the base field $F$.

(2.5) - Let $G$ be nonabelian and $p \| G \mid$. Then $\operatorname{Rad} F G$ is central if and only if $G^{\prime} P$ is a Frobenius group with kernel $G^{\prime}$, the derived group of $G$ and complement a $p$-Sylow subgroup $P$ of $G$.

We will also use the result about the dimension of the radical of $F G$ due to Brauer and Nesbitt [2]:

$$
\operatorname{dim}_{F}(\operatorname{Rad} F G)=|G|-\sum_{k=1}^{l} f_{k}^{2},
$$

where $f_{1}, \cdots, f_{l}$ are the degrees of absolutely irreducible $p$-modular representations occurring in the regular representation of $G$.

3. Relation between projective pairing property $\rho$ and the complete reducibility of induced modules for normal subgroups. As was realized by Sinha [0] and as we shall see later, the three properties under consideration are closely related to each other. In fact, they turn out to be equivalent to each other in many cases. In this section we give a proof of their equivalence for normal subgroups. The following theorem was proved by Sinha [9]. We state it and refer to the above paper for the proof.

Theorem 1. Let $H$ be a subgroup of $G$. If for eacb irreducible FH-module $\Re$, the induced FG-module $\Re^{G}$ is completely reducible then $(G, H)$ bas property $\rho$. Conversely if $H \triangleq G$ and $(G, H)$ bas property $\rho$ then $\Re^{G}$ is completely reducible for every irreducible $\mathrm{FH}-\mathrm{module} \Re$.

Remark. In particular, we always have $\mathcal{C} \subseteq \mathcal{R}$.

We now prove the following theorem.

Theorem 2. Let $H \triangleq G$. Then the following statements are equivalent:

(i) $H$ contains a p-Sylow subgroup of $G$.

(ii) $(G, H)$ is a projective pairing.

(iii) $(G, H)$ bas property $\rho$.

(iv) For every irreducible FH-module $\Re, \Re^{G}$ is completely irreducible.

Proof. The equivalence of (i) and (ii) was proved by Khatri and Sinha [7] and (iv) implies (iii) by Sinha [9]. (Note that normality of $H$ in $G$ is irrelevant for these implications.) We now prove that (ii) $\Rightarrow$ (iv) and (iii) $\Rightarrow$ (i).

(ii). $\Rightarrow$ (iv). Suppose that $H \triangleq G$ and $(G, H)$ is a projective pairing. Let $G=\bigcup_{i} H x_{i}, x_{1}=1$, be a coset decomposition of $G$ over $H$. We observe that, for $b \in H, x_{i} b=\phi_{i}(b) x_{i}$ for all $i$, where each $\phi_{i}(b)$ induces an automorphism of the 
normal subgroup $H$. We can extend each $\phi_{i}$ to an automorphism of $F H$ by linearity.

Now let $\Re$ be an irreducible $F H$-module. Then $\Re^{G}=\bigoplus \Sigma \Sigma_{i} \otimes x_{i}$ (direct sum) where each $\Re \otimes x_{i}$ is an irreducible $F H$-module. Thus $\mathfrak{R}^{G}$ is completely reducible over $F H$. Hence given any exact sequence of $F G$-modules

$$
0 \rightarrow \Re \rightarrow \Re^{G} \rightarrow L \rightarrow 0,
$$

the sequence splits over $F H$. Then by projective pairing of $(G, H)$, the sequence splits over $F G$ as well. Hence $\Re^{G}$ is completely reducible over $F G$.

Since $\Re$ was arbitrary; $H$ satisfies (iv).

(iii) $\Rightarrow$ (i). Suppose that $H \Delta G$ and $(G, H)$ has property $\rho$. Let $\mathbb{N}$ be an irreducible $F G$-module. By Clifford's theorem we have

$$
\Re_{H}=\Re_{1} \oplus \Re_{2} \oplus \cdots \oplus \Re_{l},
$$

where each $\Re_{i}$ is an irreducible $F H$-module. Consider $\Re_{1}$. We have $\operatorname{Hom}_{F H}\left(\Re_{1}, \Re_{H}\right) \simeq \operatorname{Hom}_{F G}\left(\Re_{1}^{G}, \Re\right)$ (see, for example, the proof of (43.14) in [3]). Now the left-hand side of this equation is different from zero as $\Re_{1}$ is a direct summand of $\Re_{H}$, and so is the right side. Thus $\Re$ is a composition factor of $\Re_{1}^{G}$. But by Theorem $1, \Re_{1}^{G}$ is completely reducible over $F G$. Therefore $\Re$ is a component of $\Re_{1}^{G}$ and so $\Re$ is $H$-projective ((2.1) (iii)).

As $\mathbb{N}$ was arbitrary, we infer that every irreducible $F G$-module is $H$-projective. In particular, the trivial $F G$-module $F$ is $H$-projective. Hence the vertex of $F$, namely a $p$-Sylow subgroup of $G$, is contained in $H$ (Green [4]). Thus $H$ satisfies (i).

This completes the proof of the theorem.

Corollary. Let $H \triangleq G$. Then a necessary and sufficient condition for $(G, H)$ to be a projective pairing is that every irreducible FG-module is H-projective.

To get the case on p-groups out of the way, we state the following theorem whose proof is easy, and hence omitted.

Theorem 3. Let $G$ be a p-group and $F$ a field of characteristic p. Then each of the classes $\mathcal{P}, \mathbb{R}$ and $\mathcal{C}$ is vacuous over $F$.

4. Special type of Frobenius groups. In this section we show that if $G$ is a $p$-nilpotent Frobenius group with kernel the normal $p$-complement, then the three classes $\mathcal{P}, \mathcal{R}, \mathcal{C}$ coincide. For groups of this type, Wallace's results [12], [13] are important to our investigation.

The following lemma, essentially due to Wallace [13], determines the radical of such a group.

Lemma 1. Let $G=P M, M \triangle G, P \cap M=1$, be a Frobenius group with kernel $M$ and complement a p.Sylow subgroup $P$ of $G$. Then over a field $F$ of characteristic $p$, 


$$
\operatorname{Rad} F G=\left(\sum_{x \in M} x\right) \cdot \operatorname{Rad} F P=\operatorname{Rad} F P \cdot\left(\sum_{x \in M} x\right)
$$

Proof. By Wallace's result (2.4) we have $\operatorname{dim}_{F} \operatorname{Rad} F G=|P|-1$. Let $I$ be the subspace of $F G$ spanned by $\left\{\left.\left(\Sigma_{x \in M} x\right)(g-1)\right|_{g} \in P\right\}$. Clearly $\operatorname{dim}_{F} I=|P|-1$. Since $M \triangle G$, we have

$$
y\left(\sum_{x \in M} x\right)=\sum_{x \in M} x=\left(\sum_{x \in M} x\right) y, \quad b\left(\sum_{x \in M} x\right)=\left(\sum_{x \in M} x\right) b, \quad y \in M, b \in P
$$

Therefore for $h, g \in P$ and $y \in M$, we have

$$
\text { by }\left[\left(\sum_{x \in M} x\right)(g-1)\right]=\left(\sum_{x \in M} x\right) b(g-1)=\left(\sum_{x \in M} x\right)[(b g-1)-(b-1)]
$$

and

$$
\begin{aligned}
{\left[\left(\sum_{x \in M} x\right)(g-1)\right] b y } & =\left[(g-1)\left(\sum_{x \in M} x\right)\right] b y=(g-1) b\left(\sum_{x \in M} x\right) y \\
& =[(g b-1)-(b-1)]\left(\sum_{x \in M} x\right)=\left(\sum_{x \in M} x\right)[(g b-1)-(b-1)] .
\end{aligned}
$$

Thus $I$ is an ideal in FG. But it is well known that $\operatorname{Rad} F P=\langle g-1 \mid g \in P\rangle$, hence $I=\left(\Sigma_{x \in M} x\right) \operatorname{Rad} F P$ is a nilpotent ideal of dimension $|P|-1$. This proves the lemma.

Theorem 4. Let $p$ be an odd prime and $P$ be a p.Sylow subgroup of G. If $G=P M, M \triangle G, P \cap M=1$, is a Frobenius group with kernel $M$, then the classes $\mathcal{P}, \mathcal{R}$ and $\mathcal{C}$ are equivalent.

Proof. First we observe that $G^{\prime}=M$ (Wallace [13]) so that by Wallace's result (2.5), Rad $F G \subseteq Z(F G)$.

Our scheme is to prove the inclusions $\mathscr{P} \subseteq \mathcal{C}$ and $R \subseteq \mathcal{P}$, since $\mathcal{C} \subseteq R$ always by Theorem 1 .

Suppose that $(G, H)$ is a projective pairing. We wish to show $H \in \mathcal{C}$. In the factorization $G=P M$, pick a $p$ Sylow subgroup $P$ which is contained in $H$ so that $H=P(H \cap M), H \cap M \triangle H$. Let

$$
G=\bigcup_{i} H x_{i} \text { and } M=\bigcup_{j}(H \cap M) y_{j}
$$

be coset decompositions of $G$ over $H$ and of $M$ over $H \cap M$ respectively. By Lemma 1 , a typical element $r$ of $\operatorname{Rad} F G$ can be written as 


$$
r=a\left(\sum_{x \in M} x\right)=a\left(\sum_{b \in H \cap M} b\right)\left(\sum_{j} y_{j}\right) \text {, where } a \in \operatorname{Rad} F P
$$

Let $\Re$ be an irreducible $F H$-module. Since $\operatorname{Rad} F G$ is central, we have for $r \in \operatorname{Rad} F G$,

$$
\begin{aligned}
\mathfrak{R}^{G} \cdot r & =\left(\bigoplus \sum_{i} \Re \otimes x_{i}\right) r=\bigoplus \sum_{i} \Re \otimes x_{i} r=\bigoplus \sum_{i} \Re \otimes r x_{i} \\
& =\bigoplus \sum_{i} \Re \otimes\left[a\left(\sum_{b \in H \cap M} b\right)\left(\sum_{i} y_{j}\right)\right] x_{i} \\
& =\bigoplus \sum_{i} \Re\left[a\left(\sum_{b \in H \cap M} b\right)\right] \otimes\left(\sum_{i} y_{j}\right) x_{i}=0,
\end{aligned}
$$

since $a\left(\Sigma_{b \in H \cap M} b\right) \in \operatorname{Rad} F H, H$ being a Frobenius group with kernel $H \cap M$ and $\Re$ irreducible $F H$-module. Thus $\Re^{G}$ is completely reducible over $F G$.

Since $\Re$ was arbitrary, $H \in \mathcal{C}$.

$R \subseteq \mathscr{P}$. Let $H$ be a subgroup of $G$ with $(G, H)$ having property $\rho$. Then $p$ divides $|H|$; for if not, then $F H$ is semisimple and if $\Sigma_{i} p_{i} x_{i}$ is in $\operatorname{Rad} F G$, $p_{i} \in F H, G=\bigcup_{i} H x_{i}$, coset decomposition of $G$ over $H$, then each $p_{i} \epsilon$

$\operatorname{Rad} F H=(0)$ and this implies $F G$ is semisimple, contrary to our hypothesis that $p$ divides $|G|$. In the factorization $G=P M$, pick a $p$-Sylow subgroup $P$ such that $P \cap H$ is a $p$-Sylow subgroup of $H$. Then $H=(H \cap P) \cdot(H \cap M)$ is a Frobenius group with kernel $H \cap M$ and complement $H \cap P$. Let

$$
P=\bigcup_{i}(H \cap P) x_{i} \text { and } M=\bigcup_{j}(H \cap M) y_{j}
$$

be coset decompositions of $P$ and $M$ respectively. We first show that $G=$ $\bigcup_{i, j} H y_{j} x_{i}$ is a coset decomposition of $G$ over $H$. To see this, observe that $[G: H]=[P: H \cap P][M: H \cap M]$. Suppose now that $H y_{j} x_{i}=H y_{l} x_{k}$; then

$$
\left(x_{i} x_{k}^{-1}\right)\left(x_{k} x_{i}^{-1} y_{j} x_{i} x_{k}^{-1} y_{l}^{-1}\right) \in H=(H \cap P)(H \cap M) \text {. }
$$

Therefore there exist $a \in H \cap P$ and $b \in H \cap M$ such that

$$
\left(x_{i} x_{k}^{-1}\right)\left(x_{k} x_{i}^{-1} y_{j} x_{i} x_{k}^{-1} y_{l}^{-1}\right)=a b
$$

and this gives $a^{-1}\left(x_{i} x_{k}^{-1}\right)=b\left(x_{k} x_{i}^{-1} y_{j} x_{i} x_{k}^{-1} y_{l}^{-1}\right)-1 \in P \cap M=\{1\}$. Hence $x_{i} x_{k}^{-1} \in H \cap P$ and $x_{k} x_{i}^{-1} y_{j} x_{i} x_{k}^{-1} y_{l}^{-1} \in H \cap M$. This gives $i=k$ and consequently $j=l$. Thus $\left\{H y_{j} x_{i}\right\}$ are disjoint and a cardinality argument proves our assertion.

Suppose that $(G, H)$ does not have a projective pairing. Then there exists $g \in P$ such that $g \notin H$. Write $g=b_{p} x_{0}$ where $b_{p} \in H \cap P, x_{0} \neq 1$ is in $\left\{x_{i}\right\}$. Then by Lemma 1, 


$$
r=(g-1)\left(\sum_{x \in M} x\right) \in \operatorname{Rad} F G \text {. }
$$

We now have

$$
\begin{aligned}
r & =\left(b_{p} x_{0}-1\right)\left(\sum_{x \in M} x\right)=b_{p} x_{0}\left(\sum_{x \in M} x\right)-\left(\sum_{x \in M} x\right) \\
& =b_{p}\left(\sum_{x \in M} x\right) x_{0}-\left(\sum_{x \in M} x\right), \text { since } M \Delta G, \\
& =b_{p}\left(\sum_{b \in H \cap M} b\right)\left(\sum_{j} y_{j}\right) x_{0}-\left(\sum_{b \in H \cap M} b\right)\left(\sum_{j} y_{j}\right) \\
& =\sum_{j}\left[b_{p}\left(\sum_{b \in H \cap M} b\right)\right] y_{j} x_{0}+\sum_{j}\left[-\sum_{b \in H \cap M} b\right] y_{j} .
\end{aligned}
$$

Since $\left\{y_{j} x_{i}\right\}$ are coset representatives of $G$ over $H$ and $(G, H)$ has property $\rho$, we conclude that $b_{p}\left(\Sigma_{b \in H \cap M} b\right)$ and $\Sigma_{b \in H \cap M} b$ are in $\operatorname{Rad} F H$. Now let $F$ be the trivial $H$-module. We then have $F \cdot\left(\Sigma_{b \in H \cap M} b\right)=0$. In particular, $0=1$. $\left(\Sigma_{b \in H \cap M} b\right)=\Sigma_{b \in H \cap M} 1=|H \cap M|$, which is a contradiction, since $(|H \cap M|, p)=1$.

Hence $(G, H)$ is a projective pairing and this completes the proof of the theorem.

Remark. It should be observed that for the inclusion $R \subseteq \mathcal{P}$ we only require $G$ to be $p$-nilpotent.

5. Extensions by groups of orders prime to $p$. In the last two sections we showed the equivalence of the classes $\mathcal{P}, \mathscr{R}$ and $\mathcal{C}$ for two classes of groups. We now extend the class of groups for which $\mathcal{P}=R=C$. To save space and avoid lengthy repetitions we make

Definition 5. A finite group $G$ is called a $P R C$-group over $F$ if

$$
\mathcal{P}(G, F)=\Re(G, F)=\mathcal{C}(G, F) \text {. }
$$

Trivially, if $p \nmid|G|$, then $G$ is $P R C$ over $F$; each class being all subgroups of $G$.

We start with

Lemma 2. If $H \subseteq G$, then $\operatorname{Rad} F H \subseteq \operatorname{Rad} F G$.

Proof. Let $\Re$ be an irreducible $F G$-module. Since $H \Delta G$, Clifford's theorem gives $\Re_{H}=\bigoplus \Sigma_{i} \Re_{i}$, each $\Re_{i}$ being an irreducible $F H$-module. We then have $\Re \cdot(\operatorname{Rad} F H)=\prod_{H} \cdot(\operatorname{Rad} F H)=\bigoplus \Sigma_{i} \Re(\operatorname{Rad} F H)=0$. Since $\Re$ is arbitrary, we obtain the desired result.

We next prove

Theorem 5. An extension of a PRC-group by a group with order prime to $p$ $(p=$ characteristic $F)$ is a PRC-group. 
Proof. Let $G$ be an extension of a $P R C$-group $L$ by a $p^{\prime}$-group. We wish to show $\mathscr{P}(G, F)=\mathscr{R}(G, F)=\mathcal{C}(G, F)$.

Since $\mathcal{C} \subseteq R$ always by Theorem 1 , it is sufficient to show $\mathscr{P} \subseteq \mathcal{C}$ and $\mathscr{R} \subseteq \mathscr{P}$.

$\mathcal{P}(G, F) \subseteq \mathcal{C}(G, F)$. Let $H$ be a subgroup of $G$ with $(G, H)$ a projective pairing and let $\Re$ be an irreducible $F H$-module. Then $H \supseteq P$, a $p$-Sylow subgroup of $G$; which is also a $p$-Sylow subgroup of $L$ since $[G: L]$ is prime to $p$. Since $L \Delta G$, $H \cap L \Delta H$ and so Clifford's theorem yields $\Re_{H \cap L}=\bigoplus \Sigma_{i} \Re_{i}$, where each $\Re_{i}$ is an irreducible $F(H \cap L)$-module. By the hypothesis for $L$, since $H \cap L \supseteq P=a p$ Sylow subgroup of $L$, each $\Re_{i}^{L}$ is completely reducible over $F L$. Now $L \Delta G$ and $(G, L)$ is a projective pairing. Therefore by Theorem 2 , each $\Re_{i}^{G}=\left(\Re_{i}^{L}\right)^{G}$ is completely reducible over $F G$. This shows $\left(\Re_{H \cap L}\right)^{G}=\bigoplus \Sigma_{i} \Re_{i}^{G}$ is completely reducible over $F G$.

Now $(H, H \cap L)$ is a projective pairing. Therefore, by Higman's criteria (2.1) (ii), $\Re$ is a component of $\left(\Re{ }_{H \cap L}\right)^{H}$. This in turn implies that $\Re^{G}$ is a component of the completely reducible $F G$-module $\left.\left(\Re_{H \cap L}\right)^{G}=\left(\Re_{H \cap L}\right)^{H}\right)^{G}$; thus proving its complete reducibility over $F G$. .

Since $\Re$ was arbitrary, $H \in \mathcal{C}(G, F)$.

$\mathscr{R}(G, F) \subseteq \mathscr{P}(G, F)$. Let $H$ be a subgroup of $G$ with $(G, H)$ having property $\rho$. Let $P$ be a $p$-Sylow subgroup of $G$ such that $H \cap P$ is a $p$-Sylow subgroup of H. Now $H \cap L$ is a normal subgroup of $H$ and $H \cap L \supseteq H \cap P=a$-Sylow of $H$. Therefore Theorem 2 gives property $\rho$ for the pair $(H, H \cap L)$. Since property $\rho$ is transitive and $(G, H)$ has property $\rho$ by hypothesis, consequently we get property $\rho$ for $(G, H \cap L)$.

Suppose now that $\Sigma_{i} p_{i} x_{i} \in \operatorname{Rad} F L$ with $p_{i} \in F(H \cap L)$, where $L=$ $\bigcup_{i}(H \cap L)_{x_{i}}$ is a coset decomposition of $L$ over $H \cap L$. We may also take $\left\{x_{i}\right\}$ to be some of the coset representatives of $G$ over $H \cap L$. Since $L \triangle G, \operatorname{Rad} F L$ $\subseteq \operatorname{Rad} F G$ by Lemma 2 and so $\Sigma_{i} p_{i} x_{i} \in \operatorname{Rad} F G, p_{i} \in F(H \cap L)$. By above $(G, H \cap L)$ has property $\rho$, hence each $p_{i}$ is in $\operatorname{Rad} F(H \cap L)$. This gives property $\rho$ for $(L, H \cap L)$.

By hypothesis for $L,(L, H \cap L)$ is a projective pairing. Hence $H \cap L$, and hence $H$, contains a $p$-Sylow subgroup of $L$, which is also a $p$-Sylow subgroup of G. Thus $H \in \mathscr{P}(G, F)$.

This is what we wanted to prove.

Corollary 1. If $P \Delta G$, then $\mathcal{P}(G, F)=\mathfrak{R}(G, F)=\mathcal{C}(G, F)$.

Proof. Clear from Theorem 3.

Corollary 2. If $\operatorname{Rad} F G \subseteq Z(F G)$ and $p$ is odd then $G$ is $P R C$.

Proof. By Wallace's result (2.5), $L=G^{\prime} P$ is a Frobenius group with kernel $G^{\prime}$, the derived group of $G, P$ being a $p$-Sylow subgroup of $G$. But $L$ is a PRC- 
group by Theorem 4 and $([G: L], p)=1$, so the above theorem is applicable.

Corollary 3. If $G$ is supersolvable, then $R \subseteq P$.

Proof. If $p$ is the highest prime dividing $|G|$, then $P \Delta G$ and Corollary 1 above applies. On the other hand, if $p$ is the smallest prime divisor of $G$ then $G$ is $p$-nilpotent, since it is supersolvable (Scott $[8, p .158]$ ) and $R \subseteq \mathcal{P}$ by the remark following Theorem 4. Suppose now that $p$ is an intermediate prime divisor of $|G|$. In this case there exists a normal $p$-nilpotent subgroup $K$ of $G$ (Sylow Tower Theorem, see Scott [8, p. 158]) and $G$ is an extension of $K$ by a $p^{\prime}$-group. By the remark following Theorem $4, R(K, F) \subseteq \mathscr{P}(G, F)$ by the above theorem.

We observe that using well-known results in Frobenius groups and complements (see, for example, Scott [8, p. 358]) one can obtain several types of PRC-groups. In particular we have

Corollary 4. If $|G|=p \cdot q, p, q$ being primes, or if $|G|=p q^{2}, p+q-1$, then $G$ is a PRC-group.

We omit the proof.

6. Extensions of $p$-groups. In this section we further extend the class of $P R C$ groups. In fact, we prove that an extension of a $p$-group by a $P R C$-group is a $P R C$ group.

Let $H<G$. We denote by $\mathbb{B}_{G}(H)$ the ideal in $F G$ generated by $\{b-1 \mid b \in H\}$. Similarly if $I$ is an ideal in $F G$, the set $\{g \in G \mid g-1 \in I\}$ is denoted by $\mathbb{B}_{G}^{-1}(I)$. It is easy to check that $\mathbb{B}_{G}^{-1}(I)$ is a normal subgroup of $G$. It is well known that if $\phi: F G \rightarrow F(G / H)$ is the map obtained by extending the canonical map $G \rightarrow$ $G / H(H \triangle G)$ to $F G$ by linearity, then $\operatorname{Ker} \phi=\mathbb{B}_{G}(H)$ and so $F(G / H) \cong$ $F G / \mathbb{G}_{G}(H)$.

We start with

Lemma 3. Let $P$ be a p.Sylow subgroup of G. Then

$$
B_{G}^{-1}(\operatorname{Rad} F G)=\operatorname{core}_{G}(P) \text {. }
$$

Proof. Let $A=\mathbb{B}_{G}^{-1}(\operatorname{Rad} F G)=\{g \in G \mid g-1 \in \operatorname{Rad} F G\}$. Then $A \triangle G$, since $\operatorname{Rad} F G$ is a two-sided ideal in $F G$. Since core $P \triangle G$, by Lemma 2, $\operatorname{Rad}(F($ core $P))=\langle g-1| g \in$ core $P\rangle \subseteq \operatorname{Rad} F G$. Thus for each $g \in$ core $P, g-1 \epsilon$ $\operatorname{Rad} F G$ and so core $P \subseteq A$.

Now let $g \in A$. Then $g-1$ is nilpotent, so if $H=\langle g\rangle$ then $H$ is abelian and $\operatorname{Rad} F H$ is not 0 . In such a case, it is well known that $H$ has an element in order p. Since $|H|=|g|$, so by Lagrange's theorem, $p$ divides $|g|$. Thus for each $g \in A, p$ divides $|g|$. Suppose $|A|=p^{b} m,(p, m)=1$. If $m>1$ then $A$ will have elements with orders prime to $p$, contrary to what we have proved above. Therefore 
$m=1$ and $A$ is a p-group. Since $A$ is a normal subgroup of $G$ and core $P \subseteq A$, we have $A=$ core $P$. This proves the lemma.

Theorem 6. Let $H<G$ such that $(G, H)$ bas property $\rho$. Then $H \supseteq$ core $P$.

Proof. If the assertion of the theorem is false then there is a $g \in$ core $P$ and $g \notin H$. Write $G=\bigcup_{i} H x_{i}$ : coset decomposition of $G$ over $H$. Let $g=b x_{j}$ for some $b \in H$ and some $x_{j} \neq 1$. By Lemma $3, g-1=b x_{j}-1 \cdot 1 \in \operatorname{Rad} F G$. Since $(G, H)$ has property $\rho$, we must have $b, 1 \in \operatorname{Rad} F H$, which is impossible and we are done.

Before we come to the main theorem, we need

Lemma 4. Let $I$ be a nilpotent ideal in a ring $R$ and $\phi: R \rightarrow R / I$ the natural map. If $\operatorname{Rad} R$ denotes the nilpotent radical of $R$, then $\phi(\operatorname{Rad} R)=\operatorname{Rad}(R / I)$.

Proof. We always have $\phi(\operatorname{Rad} R) \subseteq \operatorname{Rad}(R / l)$. For the reverse inclusion, suppose $a+I \in \operatorname{Rad}(R / I), a \in R$. Then for each $b+I \in R / I,(a+I)(b+I)=$ $a b+I$ is nilpotent. Thus there is an integer $m$ such that $(a b+I)^{m}=(a b)^{m}+$ $I=I$. Therefore $(a b)^{m} \in I$. Since $I$ is nilpotent by hypothesis, this yields nilpotency of $a b$. Since $b$ in $R$ was arbitrary, $a \in \operatorname{Rad} R$ and $\phi(a)=a+I \epsilon$ $\operatorname{Rad}(R / I)$. This proves the lemma.

Remark. In fact, we proved a little more than what is asserted in the statement of the lemma. We showed that $a+l$ is in $\operatorname{Rad}(R / l)$ if and only if $a$ is in $\operatorname{Rad} R$.

We are now in a position to prove the main theorem of this section.

Theorem 7. Any extension $G$ of a p-group $A$ by a PRC-group is PRC.

Proof. Once again we show $\mathcal{P} \subseteq \mathcal{C}$ and $R \subseteq \mathcal{P}$. Let $G$ be an extension of a $p$-group $A$ by a $P R C$-group. Hence $A \triangleq G$ and $G / A$ is $P R C$.

$\mathcal{P}(G, F) \subseteq \mathcal{C}(G, F)$. Let $(G, H)$ be a projective pairing. Then $H$ contains a $p$ Sylow subgroup of $G$ and hence contains $A$. Since $A \Delta H, \mathbb{B}_{A}(A) \subseteq \operatorname{Rad} F H$ by Lemma 2 and so if $\Re$ is an irreducible $F H$-module, each $a \in A$ acts trivially on $\Re$ and $\Re$ can be regarded as an irreducible $F(H / A)$-module. Now clearly $(G / A, H / A)$ is a projective pairing, hence, since $G / A$ is $P R C$, the induced $F(G / A)$-module $\Re^{G / A}=\Re \otimes_{F(H / A)} F(G / A)$ is completely reducible, over $F(G / A)$. Since $a \in A$ acts trivially on $\Re$, we can view $\Re^{G / A}$ as an $F G$-module in a natural way by defining $(n \otimes A g) \cdot b=(n \otimes A g) \cdot A b, n \in \Re, g, b \in G$.

Define $\phi: \Re^{G / A} \rightarrow \Re^{G}$ by $\phi(n \otimes A g)=n \otimes g$ and extend by linearity. Since

$$
\begin{aligned}
\phi((n \otimes A g) b) & =\phi((n \otimes A g) A b)=\phi(n \otimes A g b)=n \otimes g b \\
& =(n \otimes g) b=\phi(n \otimes A g) \cdot b
\end{aligned}
$$


and since (obviously) $\phi$ is $1-1$ and onto, $\phi$ is an FG-isomorphism.

Thus $\Re^{G}$ is completely reducible over $F G$ implying $H \in \mathcal{C}(G, F)$.

$\mathscr{R}(G, F) \subseteq \mathcal{P}(G, F)$. Suppose that $(G, H)$ has property $\rho$. Then $H \supseteq$ core $P \supseteq A$, by Theorem 6 . Let $\phi: G \rightarrow G / A$ be the natural map. We extend $\phi$ to $F G$ by linearity.

Let $G=\bigcup_{i} H x_{i}$ be a coset decomposition of $G$ over $H$. Then $G / A=$ $\bigcup_{i}(H / A) H x_{i}$ is a coset decomposition of $G / A$ over $H / A$. We show that $(G / A$, $H / A$ ) has property $\rho$.

Suppose that $\Sigma_{i} p_{i}^{\prime} A x_{i}$ is in $\operatorname{Rad} F(G / A)$, where $p_{i}^{\prime} \in F(H / A)$. There exist $p_{i} \in F H$ such that $\phi\left(p_{i}\right)=p_{i}^{\prime}$ for all $i$. Thus $\phi\left(\Sigma_{i} p_{i} x_{i}\right)=\Sigma_{i} p_{i}^{\prime} A x_{i} \in \operatorname{Rad} F(G / A)$ $=\operatorname{Rad}\left(F G / \mathscr{B}_{G}(A)\right)$. Since $\mathbb{B}_{G}(A) \subseteq \operatorname{Rad} F G$, Lemma 4 is applicable and we conclude that $\Sigma_{i} p_{i} x_{i} \in \operatorname{Rad} F G$, with $p_{i} \in F H$. By hypothesis, $(G, H)$ has property $\rho$, so each $p_{i} \in \operatorname{Rad} F H$. Thus each $p_{i}^{\prime}=\phi\left(p_{i}\right) \in \phi(\operatorname{Rad} F H)=\operatorname{Rad} F(H / A)$. This yields property $\rho$ for the pair $(G / A, H / A)$.

Now by hypothesis $G / A$ is a $P R C$-group; hence $(G / A, H / A)$ is a projective pairing and therefore $H / A$ contains a $p$-Sylow subgroup $P / A$ and $G / A, P$ being a $p$-Sylow subgroup of $G$. Thus $H \supseteq P$ and $(G, H)$ is a projective pairing.

This completes the proof of the theorem.

This gives another proof of Corollary 1 to Theorem 5 .

Corollary. If $P \triangle G$, then $G$ is $P R C$.

We observe that using the above theorem and easy known results on group theory one can prove that all groups of order $p^{2} \cdot q$ are $P R C$ without any extra conditions (contrast Corollary 4 to Theorem 5).

Remark. We do not know whether an extension of $P R C$ by $P R C$ is $P R C$ or even whether an extension of a $p^{\prime}$-group by a $p$-group is $P R C$. However, it can easily be seen that the direct product of two $P R C$-groups is a $P R C$-group.

7. Example. In the previous sections we gave some sufficient conditions for the equivalence of the three classes $\mathcal{P}, R$ and $\mathcal{C}$. That, in general, they are not equivalent is shown by the following example, which, in fact, shows that projective pairing does not imply property $\rho$. Since $\mathcal{C}$ is a subclass of $R$, this would mean that projective pairing also does not imply the complete reducibility of induced modules.

Let $G=A_{5}, H=A_{4}$ and $p=$ characteristic of $F=3$, where $A_{n}$ denotes the alternating group on $n$ symbols. Since $H$ contains a 3-Sylow subgroup of $G$, $(G, H)$ is a projective pairing over $F$. We show that $(G, H)$ cannot have property $\rho$. We may take $F$ to be a splitting field for $G$.

It is well known that over the complex field, $A_{5}$ has five irreducible representations, say, $T_{1}, T_{2}, T_{3}, T_{4}, T_{5}$ of degrees $1,2,3,4,5$ respectively. Taking 
mod 3, it follows from the results of Brauer and Nesbitt (Theorem 1 of [2] and a remark on p. 936 together with Theorem 5 of [1]) that $\bar{T}_{1}, \bar{T}_{2}, \bar{T}_{3}, \bar{T}_{4}$ remain irreducible while $\bar{T}_{5} \approx \bar{T}_{1}+\bar{T}_{4}$. Since $A_{5}$ has four 3-regular classes, these are all the irreducible representations of $A$, over the field $F$ of characteristic 3 .

Now by Brauer and Nesbitt's result (2.6) $\operatorname{dim}_{F}\left(\operatorname{Rad} F A_{S}\right)=60-$ $\left(1^{2}+3^{2}+3^{2}+4^{2}\right)=25$.

Also since $\mathrm{H}=A_{4}$ is a Frobenius group with complement a 3-Sylow subgroup of $H$, Wallace's result $(2.4)$ gives $\operatorname{dim}_{F}\left(\operatorname{Rad} F A_{4}\right)=3-1=2$. Now if $(G, H)$ has property $\rho$, then we must have $\operatorname{Rad} F G \subseteq(\operatorname{Rad} F H) F G$. This yields

$$
\operatorname{dim}_{F}(\operatorname{Rad} F G) \leq \operatorname{dim}_{F}(\operatorname{Rad} F H) \cdot[G: H] .
$$

That is, we must have $25 \leq 2 \cdot 5=10$, which is impossible. Thus $(G, H)$ cannot have property $\rho$.

Incidentally, in this case property $\rho$ does imply projective pairing; for if $(G, H)$ has property $\rho$ then 3 divides $|H|$ and since 3 occurs to the first power in $|G|=2^{2} \cdot 3 \cdot 5, H$ would contain a 3 -Sylow subgroup of $G$. This example also shows that in Theorem 5 we cannot drop the normality of $L: A_{S}$ has a subgroup $A_{4}$ with $\mathcal{P}\left(A_{4}, F\right)=\mathfrak{R}\left(A_{4}, F\right)=\mathcal{C}\left(A_{4}, F\right)$, since $A_{4}$ is Frobenius group with 3 Sylow subgroup as complement, and $\left[A_{5}: A_{4}\right]=5$ with $(3,5)=1$.

I would like to express my thanks to Professor I. N. Sinha for his many suggestions and constant encouragement during the preparation of this paper, which formed part of my $\mathrm{Ph}$. $\mathrm{D}$. thesis.

\section{BIBLIOGRAPHY}

1. Richard Brauer, Investigations on group characters, Ann. of Math. (2) 42 (1941), 936-958. MR 3, 196.

2. R. Brauer and C. Nesbitt, On the modular characters of groups, Ann. of Math. (2) 42 (1941), 556-590. MR 2, 309.

3. C. W. Curtis and I. Reiner, Representation theory of finite groups and associativealgebras, Pure and Appl. Math., vol. 11, Interscience, New York, 1962. MR 26 \#2519.

4. J. A. Green, $O n$ the indecomposable representations of finite groups, Math. Z. 70 (1958/59), 430-445. MR 24 \#A1304.

5. D. G. Higman, Modules with a group of operators, Duke Math. J. 21 (1954), 369376. MR 16, 794.

6. G. Hochschild, Relative-homological algebra, Trans. Amer. Math. Soc. 82 (1956), 246-269. MR 18, 278.

7. D. C. Khatri and I. Sinha, Projective-pairings in groups. II, Math. Japon. 14 (1969), 127-135. MR $43 \# 2121$.

8. W. R. Scott, Group theory, Prentice-Hall, Englewood Cliffs, N. J., 1964. MR $29 \# 4785$.

9. I. Sinha, On the radical of subrings of rings, Math. Student 34 (1966), 185-190. MR 38 \#3293. 
10. I. Sinha, On a converse of Clifford's theorem, Abstracts, Indian Math. Soc. 1967, 3-4.

11. - Projective-pairings in group rings, Report, Algebra Symposium, Ind. Sc. Congress, 1968.

12. D. A. R. Wallace, Note on the radical of group algebra, Proc. Cambridge Philos. Soc. 54 (1958), 128-130. MR 19, 1158.

13. - Group al gebras with central radicals, Proc. Glasgow Math. Assoc. 5 (1962), 103-108. MR 25 \#4007.

DEPARTMENT OF MATHEMATICS, MICHIGAN STATE UNIVERSITY, EAST LANSING, MICHIGAN 48823 